\title{
Aegyptio-Afroasiatica XXVII
}

\author{
Gábor Takács \\ ELTE, Hungary ${ }^{1}$
}

\section{Introduction}

During my current work on the Egyptian Etymological Word Catalogue (EEWC, ongoing since summer 1994), it has become possible to identify a great number of new lexical correspondences between Egyptian and its vast Afro-Asiatic (SemitoHamitic) kindred. The series of papers „Aegyptio-Afroasiatica” has been started in 1995 for reporting these results ${ }^{2}$. The numbering of etymological entries is continuous beginning from my very first report.

551. Eg. nn.t "eine Pflanze, aus der man Körbe macht: Binse (?)" (PT, GR, Wb II 274, 10) = "unbestimmbare Pflanze" (PT 557b, ÜKAPT III 51) = "eine Pflanze, aus der man Körbe macht: eher eine Rohr- oder Binsenart" (Keimer 1984, 29, 72.§ and p. 30, n. 6 with exx.) = "*Weide" (GHWb 416) ${ }^{3}|| \mid$ NBrb.: Shenwa i-lni $\sim$ i-lenni "roseau que l'on introduit dans l'onstou pour écarter les fils" [Laoust 1912, 146] || SBrb.: ETawllemmet tẹ̄-lèn-t "(ressemble à sensitive, graine dans gousse allongée, hauteur de la plante: 0 m. 50)" [Ncl. 1950, 41] = tệ-lən-t "espèce de roseau, jonc des mares" [Ncl. 1957, 57] = te-lăn-t, pl. telăn-en (and so also in Ayr) "1. esp. de plante (ressemble à la sensitive ou mimosa française), Cassia negricans, 2. esp. de jonc (pousse dans les mares)" [PAM 2003, 492] ||| ECh.: Somray nàlā "bamboo" [Jng. 1993 MS, 47].

552. Eg. nn.t "Name eines Vogels" (MK, Wb II 272, 8) = "kind of goose" (Davies, JEA 35, 1949, 16, nr. 10, pl. ii) = "e. Gans" (GHWb 415): perhaps to be combined with WCh.: Goemay lil "a small wild duck" [Srl. 1937, 127] || CCh.: MusguPuss liyliy (m, f) "canard siffleur" [Trn. 1991, 101]. Not clear whether Brb. *a-ylal "petit oiseau" [Ksm. 1999, 201, \#600] > NBrb.: Shilh a-ylal "oiseau de petite taille", medieval a-ylal-en (pl.) "oiseaux" [Ksm.] || SBrb.: Ahaggar (so also in

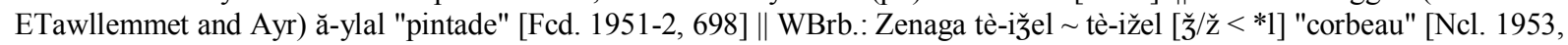
271, 401, 475] || ECu.: Tsamay lāl-o "bird sp." [Sava 2005 MS, 248] ||| WCh.: Hausa láálò "2. type of bird" [Abr. 1962, 612] are also related.

553. Eg. nn.(w)t (pl., rope or curl determinatives) "replis (?)" (CT VII 209g, AL 78.2131) = "coils (?)" (DCT 232)..$^{5}$ to be equated with NBrb.: Qabyle $\sqrt{ } \ln$ : e-ln-et and llen-tet "1. tendre les lissettes ou boucle de lisse, 2. être passé, tendu (fil de lisse, tissage sur métier de haute lisse)", i-lni, pl. i-ln-an "1. lisse (tissage), 2. ficelle" [Dlt. 1982, 457] || WCh.: Hausa lányáá "2. tying rope round and round sg.", lányàčéé "1. tied string round and round sg." [Abr. 1962, 614] < AA * $\sqrt{\ln ~ " t o ~ t i e ~ a r o u n d " ~}$ [GT]? Alternatively, cp. Sem.: MAram. (Talmud, Midrash) nīnyā? ninyā? "ein Seil aus Hanf" [Levy 1924 III 388] | Yemeni Ar. nūnah "large plait bent backwards from the crown of the head forming a loop, thereby decorating a bride's hair" [Piamenta 1990, 501] ||| LECu.: Oromo-Borana noni-ō "a rope around the muzzle of a camel during a transport" [Stroomer 1995, 212] || CCh.: Hurzo nạn "attacher" [Mch. 1953, 179] || ECh.: Sokoro nínē "binden" [Lukas 1937, 36]. Or perhaps any connection to Sem.: Hbr. lelā?ōt lālā?ōt (pl.) "knots, loops" [KB 530]? ${ }^{6}$

\footnotetext{
${ }^{1}$ The author gratefully acknowledges the support of the Hungarian Academy of Sciences for its Bolyai research fellowship facilitating the accomplishment of the present paper in the frames of the project targeting the linguistic prehistory of Ancient Egyptian.

2 A list of parts I-X with the places of publication can be found in „Aegyptio-Afroasiatica XII”, in: Lingua Posnaniensis (Poznań) 39 (1997), 93-98. Part XI was published in Acta Orientalia Acad. Scient. Hung. (Budapest) 58/4 (2005), 409-420. Part XIII appeared in Lingua Posnaniensis 45 (2003), 121-127. Part XIV in Lingua Posnaniensis 42 (2000), 151-160. Part XV in Lingua Posnaniensis (Poznań) 44 (2002), 163-174. Part XVI in Bács, T. (ed.): Studia Aegyptiaca XVII: A Tribute to Excellence, Studies Offered in Honor of Ernö Gaál, Ulrich Luft, László Török, Budapest, 2002., La Chaire d'Égyptologie, pp. 455-471. Part XVII was published in Cahiers Caribéens d'Égyptologie (Schoelcher, Martinique) 7-8 (2005), 207-235. Part XVIII appeared in Cahiers Caribéens d'Égyptologie (Schoelcher, Martinique) 5 (2003), 187-202. Part XIX was published in Rocznik Orientalistyczny (Warszawa) 57/2 (2004), 47-89. Part XX is forthcoming. Part XXI appeared in Borbone, P.G.; Tosco, M.; Mengozzi, A. (eds.): Loquentes linguis: Studi linguistici e orientali in onore di Fabrizio A. Pennacchietti, Wiesbaden, 2006., Harrassowitz Verlag, pp. 675-683. Part XXII has come out in Aula Orientalis (Barcelona) 27/2 (2009), 219-270. Part XXIII was published in Acta Orientalia Academiae Scient. Hung. (Budapest) 63/2 (2010), 223-230. Part XXIV in Bechtold, E.; Gulyás, A.; Hasznos, A. (eds.): From Illahun to Djeme: Studies Presented in Honour of Ulrich Luft, Oxford, 2011., Archaeopress, pp. 287-292. Part XXV appeared in Acta Orientalia Acad. Scient. Hung. (Budapest) 65/3 (2012), 359-372. Part XXVI, in turn, has been published in Aula Orientalis (Barcelona) 32/1 (2014), 143-149.

${ }^{3}$ This sense is corroborated by the fact that the compound hieroglyphic sign for nn represents the "two rushes with shoots" (Gardiner 1927, 471; Fischer 1983, 34, M22) = "Binse" (Staehelin 1990, 114), which - for a reason unknown to Staehelin - was coloured in the OK mostly blue, whereas in the period of Dyn. 0 and I red (three times) or black (once), cf. Kahl 1997, 51, M23. As P. Kaplony (LÄ VI 1146) rightly notes, the nn.t-plant is "(fast) identisch" with the sw.t-plant and the circumstance that "sw.t mit 5, nn.t (verdoppelt) mit 3 Stengeln schreibt, ist Konvention".

${ }_{5}^{4}$ Isolated in the Angas-Sura group (cf. Takács 2004, 230).

${ }^{5}$ Only found in CT VII 209g. As R.O. Faulkner (AECT III 103, n. 5) supposes, there is probably no connection with Eg. nn "a garment or material" (BD 340:2).

${ }^{6}$ The Hebrew word is regarded in KB 1.c. a reduplication of Sem. * $\sqrt{ }$ wy "to turn, twist" on the one hand and a cognate of Geez lelit and malelit "joint, limb, member of body etc." on the other, which is, however, impossible at a time as the latter form derives from Geez lelaya "to disjoint, separate, distinguish etc." [Lsl. 1987, 314].
} 
554. Eg. nn.wt "filet" (CT, AL 78.2132) = nn.t "fishing-net" (DCT 232) = "Netz" (GHWb 417): $:^{7}$ perhaps akin to CCh.: PMasa *1wan "net" [GT]: Masa lúwán "1. le filet sp. dit 'épervier' (pêche), 2. le filet" [Ctc. 1983, 108] = "file de pêche" [Ajl.], Marba lùwán "filet de pêche" [Ajl. 2001, 26]

555. Eg. nn "eine Speise (unter Broten und dgl. genannt)" (NE, Wb II 275, 1) = "(among pastry)" (AEO II 231*, \#535) = "*e. Brot (in e. Verkaufsliste)" (Helck: MWNR 672, §28; GHWb 416) = "food" (GR, PL 523) ||| Sem.: Ar. na?na?a "3. nourrir bien et entretenir avec soin" [BK II 1177], Yemeni nāneh "Brot" [Deboo 1989, 196] ||| LECu.: Oromo-Borana nān-ā and Wolane ńāń-ā "food, sweets" [Stroomer 1987, 372, 374; 1995, 211]. V. Orel and O. Stolbova (1989, 135; 1992, 190) combined Eg. nn with WCh.: Zaar nīn "food", which, however, cannot be derived from an earlier *nin- as they insisted.

556. Eg. nn.t (flame determinative) "Zubereitung der Milch" (XIX. Med., Wb II 272, 9) = nn (?) "Verbum zur Bez. eines Drogenzustands" (WMT 465) was correctly equated already by W. Vycichl $(1934,84)$ with WCh.: Hausa nuna "kochen" [Vcl.] = nùùná "1. to become ripe, 2. become fully cooked" [Abr. 1962, 708], which is in fact a reduplication of Ch. *nə "to ripen" $[$ Nwm. 1977, 30, \#105] $=* \sqrt{\text { ny }}(\mathrm{h})$ "mûrir" [Brt.-Jng. 1990, 172] $=* \sqrt{\text { ny/w }}$ "reifen" [Jng. 1994, 446] $=*$ nu/i-nah- "to ripen" [Stl. 1996, 83] = *n $\breve{V} / \overline{\mathrm{V}}$ - "to cook" vs. *nVh- "to ripen", *nV(nV) "to be ripe" [Stl. 2005, 100, \#339 vs. 118-9, \#425] deriving from AA $* \sqrt{n w}_{\text {nw }}$ to cook, ripen" [GT], cf. Sem.: Ar. $\bigvee_{\text {nwy }}$ II "commencer à avoir son noyau formé (dattes quand elles mûrissent)" [BK II 1374] ||| Brb. * $\bigvee_{\text {nw }}$ "to be(come) cooked, ripe" [Bynon] > e.g., NBrb.: Shilh nua "(faire) cuire, mûrir, préparer" [Jst. 1914, 145], Tazerwalt nwu "gar, reif werden" [Stumme 1899, 213], Sus nwu "être cuit, mûr" [Laoust 1921, 296] | Ntifa nwo "être cuit" [Laoust 1918, 13] | Tamazight nū "être cuit, mûr", ti-nw-it "cuisson" [Abès 1916, 131, 182] =

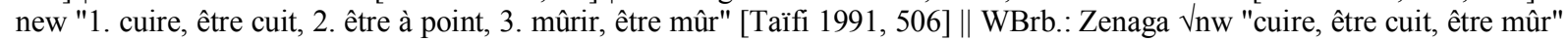
[Ncl. 1953, 222]. Cf. also Gouffé 1974, 368 (Hausa-Brb.) and Bynon 1984, 281, \#52 (Brb.-Ch.).

557. Eg. nn or nnj "le schiste ou la pierre saponaire" (Daressy, RT 30, 2) = "material of a divine statue, twice gilded, maybe a specific variety of schist of a characteristic colour (not impossible)" (Harris 1961, 89) = "schiste (?)" (Andreu \& Cauville 1977, 9; AL 77.2121): cp. perhaps CCh.: Holma nwón nwen "Stein" [Str. 1922-23, 128 and fn. 6] | Masa nón-ná "silure" [Jng. 1973 MS].

558. Eg. nn.w "Liege, Bett, Bank" (KHW after Černý) = "lit, couche" (AL), act. *lắl.`w [GT] > Cpt. (S) $\lambda \mathbf{O} \lambda$ "Bett" (KHW) = "lit, banc" (DELC): J. Osing (NBÄ 171, 682, n. 766), uncritically followed by W. Schenkel (1983, 226) and H. Satzinger

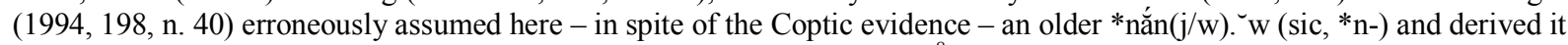
from Eg. nnj "müde sein" (MK-, Wb), which is semantically very weak. ${ }^{8}$ Osing's theory on the shift of Eg. $n . . . n>1 . . .1$ is, besides, ill-founded ${ }^{9}$ and the original $\mathrm{n}$ in Eg. nnj "tired" is clearly evidenced both internally ${ }^{10}$ and externally. ${ }^{11}$ On the top of

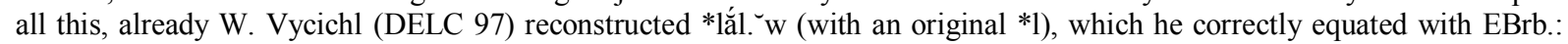
Siwa e-llal-en [ə-llal-ən] (pl.) "lit" [Lst.] = e-llal-en [Bricchetti-Robecchi] = a-lal-en [Cailliaud] = lāl-in [Stumme] = *i-lal-ěn (pl.) [Vcl.]. Further cognates appear in SBrb.: ETawllemmet ta-sə-ləl-t, Ayr te-sə-ləl-t "natte de lit (en baguettes minces d'afăzo)" [PAM 2003, 478] ||| HECu.: Sidamo lal-o (m) "wooden posts that are used transversally for the Sidamo bed" [Gsp.] $<$ AA *lal- "bed" [GT].

559. Eg. nr "to charge after" (XVIII., FD 134) = "stürmen" (GHWb 417) ${ }^{12}|| \mid$ NBrb.: Qabyle $\sqrt{ }$ nyr: neyyer (hardly Ar.) ${ }^{13}$ "continuer, poursuivre" [Dallet 1982, 588-9] ||| HECu.: Sidamo nara "to have a desire for, long for (esp. coffee, salt-beasts)" [Gsp. 1983, 245] || Ch. *nVr- "to hunt, search" [Stl. 2005, 144, \#557] > WCh.: Saya nar "hunting" [Krf.] = naar "a hunt" [Csp.] || CCh.: Buduma nera "suchen" [Nachtigal apud Lukas 1939, 120].

\footnotetext{
${ }^{7}$ Its inner Egyptian etymologies are unsuccessful. R. O. Faulkner (AECT II 119-120, spell 477, n. 3-4), for instance, rendered nn.wt in CT VI $34 \mathrm{i}$ as "dead-net" identifying it with nn.wt "dead" and considering the sense "net" as a result of a simplication from, a brief form of a compound attested in CT VI $34 \mathrm{f}$ as h. $3 \mathrm{~m}-\mathrm{nnnn} . \mathrm{t}$ (corruption for -nn.wt) "the net for catching the dead". J. Osing (NBÄ 733, n. 885), in turn,

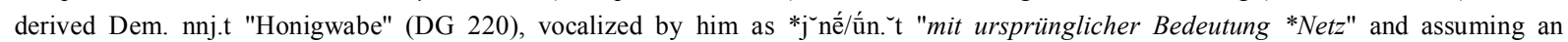
"Apokope der Vortonsilbe $*{ }^{\mathrm{j}}{ }^{-}->* *^{-}-"$, from Eg. jnn "1. jem. (etw.) umwenden, 2. mit hrr: etw. von jem. abwenden, fortbringen" (PT-, Wb I 97, 7-8), whose basic meaning he forged to have been "*umwinden", whic is nowhere attested and does not even issue from the attested senses.

${ }^{8}$ The arguments for an Eg. hapax nnj "ermattet daliegen" (once in a NK tomb inscription) are very weak, cf. sw m nn r` nb "(der des Nachts nicht schlief) er liegt alle Tage ermattet da" (Osing after Kees, ZÄS 62, 1926, 76), which can also be translated with the usual sense of nnj: "he is tired every day".

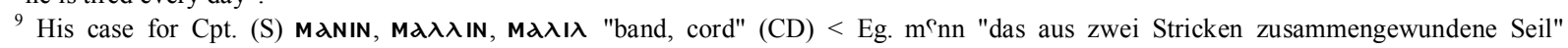
(Pfortenbuch, $\mathrm{Wb})=$ "das gewundene Seil" (Hornung) = "twisted rope" $(\mathrm{CED})=$ "der Doppeltgewundene" (Zeidler) = "Schlangenseil, aus dessen Windungen die Stunden entstehen" (LÄ VI 1363) = „Seil (aus zwei Stricken zusammengewunden)” (GHWb), being an ex. of a nasal dissimiliation of $\mathrm{m} . . . \mathrm{n}>\mathrm{m} . . .1$, proves little about $(\mathrm{S}) \boldsymbol{\lambda} \mathbf{0} \boldsymbol{\lambda}$.

${ }^{10}$ Cf. Cpt. (O) N€nOY[T] = "Ermatten, Mattigkeit" (1x: pap. BM 10808, 1. 8, Osing 1976, 20, 66-67, NBÄ 85)<*n`nwát=.

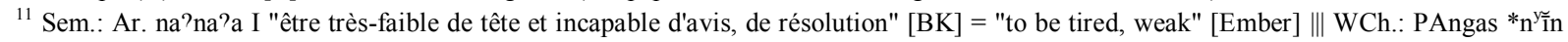
„tired” [GT]: Angas nyin „(to be) tired out, fatigued” [Flk.] = nyiin [Jng.] = nyin [n $\left.\mathrm{n}^{\mathrm{y}} \mathrm{in}\right]$ [Brq.] = nyiin [Hfm.] = nyin [ALC] = nyìn [Krf.] $\|$ ECh.: WDangla núún "sans force" [Fédry], Mokilko nùuné (m) "(un) mort, cadavre" [Jng.]. For the Eg.-Ar. compariosn see Ember 1913, 118, \#72; ESS §11.a.25. In the Mokilko cognate we can see perfectly the same shift of meaning as in Eg. nnj.w "die Müden als Bez. für gespenstische Tote" (ME, Wb).

${ }^{12}$ Compared by Ch. Ehret $(1995,333, \# 651)$ with Ar. $\sqrt{ }$ nrš "to reach for, take in one's hand" and a certain ECu. *nawr- "to arrive, come up to" (one of Ehret's ECu. reconstructions that are much less reliable than those by H.-J. Sasse 1979), which semantically do not fit.

${ }^{13}$ No Arabic root appears it might be explained from. BK II 1375 only has $\sqrt{ }$ nyr IV "2. appeler qqn. tout haut".
} 
560. Eg. nrj (IIIae inf.) "erschrecken, schaudern, schrecklich sein" (PT-, Wb II 277, 4-8) = "to be terrified, shudder" (Breasted 1930, 143-144), also intr. "to be afraid" (PT, Allen 1984, 570): its etymology has been surrounded by controversies as usually in Egyptian. It was treated by W. Westendorf $(1962,11$, §22.c.5) as a mere root variety of Eg. nwr 3wr "zittern", which is semantically certainly not tenable. Not much better is the suggestion by E. Zyhlarz $(1934,119)$, who equated it with Tuareg ẹ-nrú $\sim$ ẹ-rnú "obsiegen, übermächtig sein", but the $\mathrm{SBrb}$. root was $* \sqrt{ } \mathrm{rnC}_{3}\left(\mathrm{C}_{3}=\right.$ weak consonant?) with a basic meaning "to win, subdue" [GT]. ${ }^{14}$ Let alone A.R. Bomhard (1981, 450; 1984, 277, \#295), who combined the Eg. root directly with IE *nə/ar- "to be strong, manly". The true cognates of Eg. nrj appear in Sem.: Hbr. $\sqrt{ }$ n? $\mathrm{r}$ "to be abhorred, spurned"

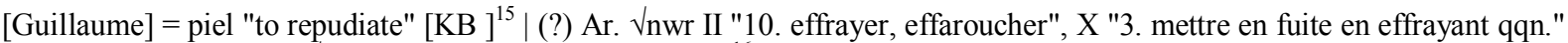
[BK II 1364-5] || Ch. * $\sqrt{n r}$ "(to) fear" [JS 1981, 101, C $]^{16}>$ CCh.: Masa náárá "craindre", nààrà "peur", náár-dá "le peur" [Jng. 1973 MS] = ñār "1. la peur, 2. (adj.) qui a peur, 3. le pereux, le lâche" [Ctc. 1983, 121] || ECh.: Kwang-Mobu nāāré "to fear" [Jng. 1972 MS, 22, \#569] = -Mobu naare vs. -Ngam naaré "craindre, avoir peur" [Lns. 1982, 109; 1984, 69], KwangGaya tê-ñ Ar and -Alowa tê-ñar and -Mindera ti-nAr "avoir peur" [Coates 1991 MS, 3]. Since Eg. nr.w "der Schrecken, Respekt" (PT-, Wb II 277-8), i.e., *nî́r. ${ }^{-w}$ (Schenkel 1983, 226; Kahl 1994, 78), ${ }^{17}$ contained not just a transliterational, but a "real" [-r-], ${ }^{18}$ the alternative AA etymology (another AA root for "to fear" with *-1-) ${ }^{19}$ is to be ruled out.

561. Eg. * $\sqrt{\text { nrj }}>$ nr.wt "Art Bruch (der Rückenwirbel und der Rippen)" (Med., Wb II 279, 8-9) = "an injury producing a rending or strain of an articulation without a dislocation or a break: a sprain (in a vertebra of neck)" (Breasted 1930, 320-322) = "Zerrung (als Knochengelenkverletzung)" (GHWb 418; WMT 467) = "sprain or joint injury" (Nunn 1996, 223): finding it "difficult to trace nr.wt to any known root", J. H. Breasted (1930, 323), nevertheless, could not resist the temptation of falling into a speculation that "it has evidently (!) inclined in its semantic history in a different direction from nr.t 'fright, terror' ... or nr.t 'neck' (of collar bone) ...", for either of which, however, he failed to present a convincing argument. Instead, there is no serious obstacle in the way of combining Eg. nr.wt with ECu.: Dullay: Harso and Dobase nar- "zusammenfallen einstürzen (altes Haus, Stuhl, Baum u.ä.)" [AMS 1980, 176] $]^{20}|| \mid$ ECh.: EDangla nòrìyē "dissoudre, désintégrer, désagréger" [Djibrine \& Montgolfier 1973, 220] | Mawa nàr-án (verbal noun suffix -n) "zerstören" [Jng. 1978, 38] = nó nárê "ich zerstöre" [Jng. 1981, 56].

562. Eg. nrj "bestimmter Zeitpunkt, bes. beim Ackerbau" (NK, Wb II 279, 11-13) = "specified time, term" (CED 105 pace $\mathrm{Wb}$ ) = "time, the return of the year" (AEO I 12*, nr. 57; Caminos 1954 LEM, 380f.; 1956, 33-34; FD 135) = "das wiederkehrende Jahr (nicht einfach Zeit, wie noch Gardiner dachte)" (Guglielmi 1973, 155), nr.t "year", (m) nr.t-nr.t "year after year" (Blackman \& Fairman, JEA 29, 1943, 23f.) ||| HECu. *nir- "year, last" [Hudson 1989, 419]. With regard to Cpt. (B) N€I, NHI, (L) N€€ (f) "Termin, Zeit(punkt)" (KHW 120; Vergote 1950, 293) = "délai, terme, instant" (DELC 141) < Dem. n3j "Zeit" (DG 205:5) vs. nj3 "term, timespan" (Smith 1987, 171) < *nĕ́rjॅ(w/j) (NBÄ 432) = *nirj.at $\rightarrow$ *ni3j.at *niy3.at (DELC), in which *-e/i-, besides, corroborates the correctness of its equation with HECu. *nir-, one can hardly agree with V. Orel and O. Stolbova (1992, 189; HSED \#1665) to affiliate Eg. nrj with CCh.: Matakam leř and Banana lor "time".

\section{Abbreviations of languages}

(A): Akhmimic, AA: Afro-Asiatic, Akk.: Akkadian, Ar.: Arabic, Aram.: Aramaic, (B): Bohairic, Bed.: Bed'awye, Brb.: Berber, Ch.: Chadic, CCh.: Central Chadic, CT: coffin texts, Cu.: Cushitic, ECh.: East Chadic, ECu.: East Cushitic, E: East(ern), Eg.: Egyptian, EWlmt.: East Tawllemmet, (F): Fayyumic, GR: Greek (Ptolemaic) and Roman Period, GW: syllabic or group-writing, HECu.: Highland East Cushitic, IMP: Intermediate Period, JAram.: Jewish Aramaic, (L): Lycopolitan (or Subakhmimic), LECu.: Lowland East Cushitic, Lit.: literary texts, LP: Late Period, M: Middle, MK: Middle Kingdom, N: North, NBch.: North Bauchi, NBrb.: North Berber, NK: New Kingdom, NOm.: North Omotic, OEg.: Old Egyptian, OK: Old Kingdom, Om.: Omotic, PCh.: Proto-Chadic, PCu.: Proto-Cushitic, PT: pyramid texts, S: South(ern), (S): Sahidic, SBrb.: South Berber, Sem.: Semitic, W: West(ern), WBrb.: West Berber, WCh.: West Chadic, WSem.: West Semitic.

Abbreviations of author names

\footnotetext{
${ }^{14}$ Cf. Ahaggar e-rnu "vaincre" [Fcd. 1951-2, 1643], ETawllemmet and Ayr a-rṇu "vaincre, subjuger etc." [PAM 2003, 674].

${ }^{15}$ The etymology of this Hebrew root, rendered in GB 478 still as "(ein Heiligtum) entweihen, (einen Bund) aufheben", has always been

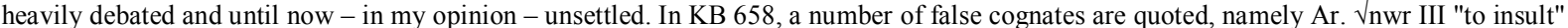
(after König), Hbr. $\sqrt{ } \mathrm{n} \odot \mathrm{r}$ "to roar" (after Driver), held to be a dialectal by-form of Hbr. $\sqrt{ }$ ? $\mathrm{rr}$ "to curse" (KB). A. Guillaume (1965 IV 9), in turn, equated it with Ar. ma?ira "to be hated". E. Klein $(1987,401)$ suggested a comparison with Akk. nâru nêru G "(er)schlagen, töten" [AHW 780], which is certainly out of the question the underlying Semitic root being *Vnhr "durchstechen, -bohren" [AHW].

${ }^{16}$ The comparison of Eg. nrj with C/ECh. *nār "to fear" (without mentioning reflexes in the Chadic daughter languages, just with a remark: "reconstruction error?" for an expected *nar) was first put forward by Ch. Ehret $(1995,331, \# 646)$ along with two further uncertain parallels: Ar. $\sqrt{ }$ rrz "to hide o'self for fear" and SCu.: Qwadza nyele-siko "thing of supernatural danger".

${ }_{17}$ M. Görg (VT 35, 1985, 363-368, cf. AEB 85.328) explained, by the way, Hbr. nīr "ein 'Machtzeichen' Davids" (in 1 Kings XI 36), which, by the way, is traditionally rendered "Licht, Leuchte, bildl. v. Fortbestehn eines Geschlechtes" [GB 503] = "light, lamp" [KB 697], from Akk. niru "1. Joch, Querholz, 2. Joch der Herrschaft" [AHW 793-4] = "dominion" [Görg] as well as Eg. nr.w "terror".

${ }^{18}$ This [-r-] is reflected in the personal name transcribed as Ảvípıৎ reflecting Eg. ${ }^{3} 3$-nr.w, cf. NBÄ 85.

${ }^{19}$ Nbrb.: Tamazight nnul "1. craindre, 2. s'enfaire, s'inquiéter, se soucier" [Taïfi 1991, 490] || ECh. *لVln- "peur" [JI]: Tumak lān "craindre, avoir peur" [Caprile 1975, 79], Gadang láány "avoir peur" [JI 1990 MS, 1, \#17] | Somray laińe "fürchten" [Lukas 1937, 80] = lány "avoir peur", lányá (m) "peur" [Jng. 1993 MS, 41].

${ }^{20}$ This word was identified by Ch. Ehret $(1991,268$, \#242) with LECu.: Arbore nar-ḍ-os "to make thin, emaciate (tr.)" etc. < ECu. *ja/ir- "to be weak, frail", which is semantically unconvincing.
} 
Abr.: Abraham, Ajl.: Ajello, AMS: Amborn, Minker, Sasse, BK: Biberstein Kazimirsky, Bmh.: Bomhard, Brt.: Barreteau, Cpr.: Caprile, Ctc.: Caïtucoli, Dlt.: Dallet, DM: Djibrine \& Montgolfier, Fcd.: Foucauld, GB: Gesenius \& Buhl, Grd.: Gardiner, Gsp.: Gasparini, GT: Takács, Hds.: Hudson, JI: Jungraithmayr and Ibriszimow, Jng.: Jungraithmayr, JS: Jungraithmayr and Shimizu, Jst.: Justinard, KB: Koeehler \& Baumgartner, Krf.: Kraft, Ksm.: Kossmann, Lks.: Lukas, Lns.: Lenssen, Lsl.: Leslau, Lst.: Laoust, Mch.: Mouchet, Ncl.: Nicolas, Nwm.: Newman, OS: Orel \& Stolbova, PAM: Prasse, Alojaly, Mohamed, Snk.: Schenkel, Srl.: Sirlinger, Ss.: Sasse, Stl.: Stolbova, Str.: Strümpell, Strm.: Stroomer, Stz.: Satzinger, Tf.: Taïfi, Trn.: Tourneux, Vcl.: Vycichl, Vrg.: Vergote, Wst.: Westendorf, Zhl.: Zyhlarz.

Quoted works

Abès, M.: Manuel de berbère marocain. (Place not indicated), 1916. (?), (publisher not indicated). Abraham, R. C.: Dictionary of the Hausa Language. ${ }^{2}$ London, 1962., University of London Press. $\mathrm{AEB}=$ Annual Egyptological Bibliography. AECT $=$ Faulkner, R. O.: The Ancient Egyptian Coffin Texts. Vol. I-III. Warminster, 1973-8., Aris \& Phillips Ltd. AEO = Gardiner, A. H.: Ancient Egyptian Onomastica. I-II. Oxford, 1947., Clarendon Press. AHW = Soden, W. von: Akkadisches Handwörterbuch. I-III. Wiesbaden, 1965-1981., Otto Harrassowitz. Ajello, R.; Karyo, M.; Melis, A.; Dobio, Ou.: Lexique comparatif de six langues tchadique central (Gizey, Ham, Lew, Marba, Masa, Musey). Pisa, 2001., Edizioni Plus, Università di Pisa.

AL I-III = Meeks, D.: Année lexicographique. Égypte ancienne. Tome 1-3 (1977-1979). $2^{\text {ème }}$ édition. Paris, 1998.,

Cybele.

Allen, T.G.: The Inflection of the Verb in the Pyramid Texts. Bibliotheca Aegyptia, vol. 2, fasc. 1-2. Malibu, 1984., Undena Publications.

Amborn, H. \& Minker, G. \& Sasse, H.-J.: Das Dullay. Materialen zu einer ostkuschitischen Sprachgruppe. Berlin, 1980., Reimer Verlag.

Andreu, G. \& Cauville, S.: Vocabulaire absent du Wörterbuch (I).= Revue d'Égyptologie 29 (1977), 5-13.

Barreteau, D. \& Jungraithmayr, H.: Les verbes monoradicaux dans les langues tchadiques.= Jungraithmayr, H. \& Tourneux, H. (eds.): Études tchadiques. Verbes monoradicaux suivis d'une note sur la negation en haoussa. Actes de la XIIème réunion de Groupe d'Études Tchadiques LACITO-CNRS-PARIS. Paris, 1990., Librairie Orientaliste Paul Geuthner. Pp. 37-214.

Biberstein Kazimirski, A. de: Dictionnaire arabe-français. Paris, 1860., Maisonneuve \& Co. Editeurs.

Bomhard, A. R.: Indo-European and Afroasiatic. New Evidence for the Connection.= Arbeitman, Y. L. \& Bomhard, A. R. (eds.): Bono Homini Donum. Essays in Historical Linguistics in Memory of J. Alexander Kerns. Amsterdam, Philadelphia, 1981., John Benjamins. Pp. 351-474.

Bomhard, A. R.: Toward Proto-Nostratic. Amsterdam, 1984., John Benjamins.

Breasted, J.H.: The Edwin Smith Surgical Papyrus. Vol. I. Chicago, 1930., The University of Chicago Press.

Bynon, J.: Berber and Chadic. The Lexical Evidence.= Bynon, J. (ed.): Current Progress in Afro-Asiatic Linguistics. Amsterdam, Philadelphia, 1984., John Benjamins. Pp. 241-290.

Caïtucoli, C.: Lexique masa. Paris, 1983., Agence de Coopération Culturelle et Technique. Caminos, R.: Late-Egyptian Miscellanies. London, 1954., Oxford University Press.

Caminos, R.: Literary Fragments in the Hieratic Script. Oxford, 1956., Oxford University Press. Caprile, J.-P.: Lexique tumak-français (Tchad). Berlin, 1975., Verlag von Dietrich Reimer. CD = Crum, W. E.: A Coptic Dictionary. Oxford, 1939., Oxford, 1939., Oxford University Press. $\mathrm{CED}=$ Černý, J.: Coptic Etymological Dictionary. London, Cambridge, 1976., Cambridge University Press. Coates, (?): Kwang Wordlists. MS. 1991. 6 p.

CT = Buck, A. de: The Egyptian Coffin Texts. Vol. I-VII. Chicago, 1935-61., The University of Chicago Press. Dallet, J.-M.: Dictionnaire qabyle-français. Parler des At Mangellat (Algerie). Paris, 1982., SELAF (Société d'études linguistiques et anthropologiques de France).

DCT $=$ Molen, R. van der: A Hieroglyphic Dictionary of Egyptian Coffin Texts. Leiden, 2000., E.J. Brill.

Deboo, J.: Jemenitisches Wörterbuch. Arabisch-Deutsch-Englisch. Wiesbaden, 1989., Harrassowitz.

DELC $=$ Vycichl, W.: Dictionnaire étymologique de la langue copte. Leuven, 1983., Peeters.

DG $=$ Erichsen, W.: Demotisches Glossar. Koppenhagen, 1954., Ejnar Munksgaard.

Djibrine, B. A. Z. \& Montgolfier, P. de (etc.): Vocabulaire dangaléat. Kawo dayla. Place not indicated, around 1973. (deduced by G. Takács), publisher not indicated.

Ehret, Ch.: The Historical Reconstruction of Southern Cushitic Phonology and Vocabulary. Berlin, 1980., Dietrich

Reimer Verlag.

Ehret, Ch.: The Consonant Inventory of Proto-Eastern Cushitic.= Studies in African Linguistics 22/3 (1991), 211-

275.

Ehret, Ch.: Reconstructing Proto-Afroasiatic (Proto-Afrasian). Vowels, Tone, Consonants, and Vocabulary.

Berkeley, Los Angeles, California, 1995., University of California.

Ember, A.: Kindred Semito-Egyptian Words. New Series.=ZÄS 51 (1913), 110-121.

ESS = Ember, A.: Egypto-Semitic Studies. Leipzig, 1930., The Alexander Cohut Memorial Foundation.

FD = Faulkner, R. O.: A Concise Dictionary of Middle Egyptian. Oxford, 1962., Clarendon Press.

Fischer, H. G.: Ancient Egyptian Calligraphy. $2^{\text {nd }}$ edition. Now York, 1983., The Metropolitan Museum of Art.

Foucauld, Ch. de: Dictionnaire touareg-français, dialecte de l'Ahaggar. Vol. I-IV. Paris, 1951-52., Imprimerie Nationale de France.

Gardiner, A. H.: Egyptian Grammar. ${ }^{1}$ Oxford, 1927., Clarendon Press.

Gasparini, A.: Sidamo-English Dictionary. Bologna, 1983., E.M.I. 
GB = Gesenius, W. (bearbeitet von Buhl, F.): Hebräisches und aramäisches Handwörterbuch über das Alte Testment. Unveränderter Neudruck der 1915 erschienenen 17. Auflage. Berlin, Göttingen, Heidelberg, 1962., SpringerVerlag.

GHWb = Hannig, R.: Grosses Handwörterbuch Ägyptisch-Deutsch (2800-950 v. Chr.). Mainz, 1995., Verlag Philipp von Zabern.

Gouffé, C.: Contacts de vocabulaire entre le haoussa et le touareg.= Cohen, D. (ed.): Actes du Premier Congrés International de Linguistique Sémitique et Chamito-Sémitique, Paris, 16-19 juillet 1969. Paris, 1974., Mouton. Pp. 357-380.

Guglielmi, W.: Reden, Rufe und Lieder auf altägyptischen Darstellungen der Landwirtschaft, Viehzucht, des Fischund Vogelfangs vom Mittleren Reich bis zur Spätzeit. Bonn, 1973., Rudolf Habelt Verlag.

Guillaume, A.: Hebrew and Arabic Lexicography. A Comparative Study. Reprinted from: Abd-Nahraim. Vol. I-IV (1959-65). Leiden, 1965., Brill.

Harris, J.R.: Lexicographical Studies in Ancient Egyptian Minerals. Berlin, 1961., Akademie-Verlag.

HSED = Orel, V. É. \& Stolbova, O. V.: Hamito-Semitic Etymological Dictionary. Leiden, 1995., E. J. Brill.

Hudson, G.: Highland East Cushitic Dictionary. Hamburg, 1989., Buske.

$\mathrm{JEA}=$ Journal of Egyptian Archaeology (London).

Jungraithmayr, H.: Wordlist of Kwang-Mobu. 10-17 Jan. 1972. MS. 32 p.

Jungraithmayr, H.: Masa (Bongor) Lexicon. MS. Marburg (now in Frankfurt), 1973.

Jungraithmayr, H.: Mawa. MS. 21 February 1978. 65 p.

Jungraithmayr, H. \& Shimizu, K.: Chadic Lexical Roots. Vol. II. Tentative Reconstruction, Grading and Distribution. Berlin, 1981., Verlag von Dietrich Reimer.

Jungraithmayr, H.: Über die Mawa (Guera, Tschad) - Ethnographische und linguistische Notizen.= Hofmann, I.

(Hrsg.): Festschrift zum 60. Geburtstag von P. Anton Vorbichler. I. Teil. Wien, 1981., Institut für AFrikanistik und Ägyptologie der Universität Wien. Pp. 47-70. 1990. $15 \mathrm{p}$ Jungraithmayr, H. \& Ibriszimow, D.: Wordlist of Sarwa, Gadang, Miltu (Gali). MS. Groupe d'Études Tchadiques.

Jungraithmayr, H. \& Ibriszimow, D.: Sur les langues sarwa, gadang et miltu (groupe sibine/somray). MS. Groupe d'Études Tchadiques. 1990. 20 p.

Jungraithmayr, H.: Lexique sibine (sumray)-français. MS. Frankfurt a/M, versions of 20 April 1993 ( $\vee$

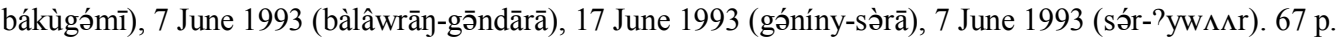

Jungraithmayr, H.: Zum Stand der Erforschung alttschadischen Wortgutes.= Wunsch, C. (ed.): XXV. Deutscher

Orientalistentag, Vorträge, München, 8.-13.4.1991. Stuttgart, 1994., Franz Steiner Verlag. Pp. 443-452.

Justinard, (?): Manuel de berbère marocain (dialecte chleuh). Paris, 1914., Librairie Orientaliste Paul Geuthner.

Kahl, J.: Das System der ägyptischen Hieroglyphenschrift in der 0.-3. Dynastie. Wiesbaden, 1994., Harrassowitz

Verlag.

Kahl, J.: Die Farbgebung in der frühen Hieroglyphenschrift.= ZÄS 124 (1997), 44-56.

$\mathrm{KB}=$ Koehler, L. \& Baumgartner, W.: The Hebrew and Aramaic Lexicon of the Old Testament. I-V. Leiden, 1994-

2000, E. J. Brill.

Zabern.

Keimer, L.: Die Gartenpflanzen im Alten Ägypten. Band II. Hrsg. von Renate Germer. Mainz, 1984., Philipp von

KHW $=$ Westendorf, W.: Koptisches Handwörterbuch. Heidelberg, 1977., Carl Winter Universitätsverlag.

Klein, E.: A Comprehensive Eymological Dictionary of the Hebrew Language for Readers of English. New York,

1987., Macmillan.

Kossmann, M.: Essai sur la phonologie du proto-berbère. Köln, 1999., Köppe

Kraft, Ch. H.: Chadic Wordlists. I-III. Berlin, 1981., Dietrich Reimer Verlag.

Laoust, E.: Étude sur le dialecte berbère du chenoua comparé avec ceux des Beni-Menacer et des Beni-Salah. Paris,

1912., Ernest Leroux.

Laoust, E.: Étude sur le dialecte berbère des Ntifa. Grammaire. Textes. Paris, 1918., Ernest Leroux.

Laoust, E.: Cours de berbère marocain. Grammaire - vocabulaire - textes. Dialectes du sous du Haut et de l'Antiatlas. Paris, 1921., Augustin Challamel.

LÄ = Helck, W. \& Westendorf, W. (Hrsg., begründet von W. Helck und E. Otto): Lexikon der Ägyptologie. Band

I-VII. Wiesbaden, 1975-92., Harrassowitz.

Lenssen, T.: Das Verb im Kwang (Tschad) - eine phonologische Studie. M.A. thesis, Marburg, 1982., Philipps-

Universität Marburg.

Lenssen, T.: Studien zum Verb im Kwang (Tschad).= Africana Marburgensia Sonderheft 8 (1984).

Leslau, W.: Comparative Dictionary of Ge ${ }^{\mathrm{e}} \mathrm{z}$ (Classical Ethiopic). Wiesbaden, 1987., Otto Harrassowitz.

Levy, J.: Wörterbuch über die Talmudim und Midraschim nebst Beiträgen von Heinrich Leberecht Fleischer.

Zweite Auflage mit Nachträgen und Berichtigungen von Lazarus Goldschmidt. I-IV. Berlin \& Wien, 1924., Benjamin Harz Verlag.

Lukas, J.: Zentralsudanische Studien.= Abhandlungen aus dem Gebiet der Auslandskunde. Hansische Universität, Reihe B, Band 45/24 (1937).

Lukas, J.: Die Sprache des Buduma in Zentralen Sudan.= Abhandlungen für die Kunde des Morgenlandes 24/2

Mouchet, J.: Vocabulaires comparatifs de sept parlers du Nord-Cameroun.= Bulletin de la Société d'Études Camerounaises 41-42 (1953), 136-206.

MWNR = Helck, W.: Materialien zur Wirtschaftsgeschichte des Neuen Reiches. Teil II.= Akademie der Wissenschaften und der Literatur, Abhandlungen der Geistes- und Sozialwissenschaftlichen Klasse 11 (1960). Teil III: 2 (1963). Teil IV: 3 (1963). Teil V: 4 (1964). Teil VI: 4 (1969). With continuous pagination. 
NBÄ = Osing, J.: Die Nominalbildung des Ägyptischen. I-II. Maiz/Rhein, 1976., Verlag Philipp von Zabern.

Newman, P.: Chadic Classification and Reconstructions.= Afroasiatic Linguistics 5/1 (1977), 1-42.

Nicolas, F.: Tamesna. Les Ioullemmeden de l'Est ou Touâreg "Kel Dinnîk". Cercle de T'âwa - Colonie du Niger. Notes de linguistique et d'éthnographie berbères. Dialectes de la Tamàžəq-Taulləmét. Paris, 1950., Imprimerie Nationale. Nicolas, F.: La langue berbère de Mauritanie. Dakar, 1953., Institut Français d'Afrique Noire.

Nicolas, F.: Vocabulaires ethnographiques de la Tamâjeq des Iullemmeden de l'est (Touâreg de la Colonie du Niger, Afrique Occidentale Française).= Anthropos 52 (1957), 49-63, 564-580.

Nunn, J.: Review of Walker, J.H.: Studies in Ancient Egyptian Anatomical Terminology.= JEA 85 (1999), 257-

Orel, V. É. \& Stolbova, O. V.: Čadsko-egipetskie izoglossy v oblasti kul'turnoj leksiki.= Lingvističeskaja rekonstrukcija i drevnejšaja istorija Vostoka. Čast' 1. Moskva, 1989., Nauka. Pp. 131-136.

Orel, V. É. \& Stolbova, O. V.: On Chadic-Egyptian Lexical Relations.= Shevoroshkin, V. (ed.): Nostratic, DeneCaucasian, Austric and Amerind. Bochum, 1992., Brockmeyer. Pp. 181-203.

Osing, J.: Der spätägyptische Papyrus BM 10808. Wiesbaden, 1976., Harrassowitz.

Piamenta, M.: Dictionary of Post-Classical Yemeni Arabic. I-II. Leiden, 1990-91., Brill.

1997., Peeters.

$\mathrm{PL}=$ Wilson, P.: A Ptolemaic Lexikon. A Lexicographical Study of the Texts in the Temple of Edfu. Leuven,

Prasse, K.-G.; Alojaly, Gh.; Mohamed, Gh.: Dictionnaire touareg-français (Niger). Copenhagen, 2003., Museum Tusculanum Press, University of Copenhagen.

PT = Sethe, K.: Die altägyptischen Pyramidentexte. I-II. Leipzig, 1908., 1910., J.C. Hinrichs'sche Buchhandlung.

RT = Recueil de Travaux Relatifs à la Philologie et à l'Archéologie Égyptiennes et Assyriennes (Paris).

Sasse, H.-J.: The Consonant Phonemes of Proto-East-Cushitic (PEC).= Afroasiatic Linguistics 7/1 (1979), 1-67.

Satzinger, H.: Das ägyptische «Aleph»-Phonem.= Zwischen den beiden Ewigkeiten. Festschrift Gertrud Thausing.

Wien, 1994., Eigenverlag des Institutes für Ägyptologie der Universität Wien. Pp. 191-205.

Savà, G.: English-Ts'amakko Wordlist. MS. Leiden, 2005.

Harrassowitz

Schenkel, W.: Zur Rekonstruktion der deverbalen Nominalbildung des Ägyptischen. Wiesbaden, 1983.,

Sirlinger, E.: Dictionary of the Goemay Language. Jos, Nigeria, 1937., Prefecture Apostolic of Jos.

Smith, M.: Mortuary Texts of Papyrus BM 10507. London, 1987., British Museum Publications Ltd.

Staehelin, E.: Zu den Farben der Hieroglyphen.= Hornung, E. (Hrsg.): Zwei ramessidische Königsgräber: Ramses

IV. und Ramses VII. Main am Rhein, 1990., Philipp von Zabern. Pp. 101-119.

Stolbova, O. V.: Studies in Chadic Comparative Phonology. Moscow, 1996., "Diaphragma" Publishers.

Stolbova, O. V.: Chadic Lexical Database. Issue I. L, N, NY, R. Kaluga, 2005., Poligrafiya.

Stroomer, H.: A Comparative Study of Three Southern Oromo Dialects in Kenya. Hamburg, 1987., Buske.

Stroomer, H.: A Grammar of Boraana Oromo (Kenya). Phonology, Morphology, Vocabularies. Köln, 1995., Buske.

Strümpell, F.: Wörterverzeichnis der Heidensprachen des Mandara-Gebirges (Adamaua).= Zeitschrift für Eingeborenen-Sprachen 13 (1922-23), 109-149.

Stumme, H.: Handbuch des Schilchischen von Tazerwalt. Leipzig, 1899., J. C. Hinrichs'sche Buchhandlung.

Taïfi, M.: Dictionnaire tamazight-français (parlers du Maroc central). Paris, 1991., L'Harmattan-Awal.

Takács, G.: Comparative Dictionary of the Angas-Sura Languages. Berlin, 2004., Dietrich Reimer Verlag. Geuthner.

Tourneux, H.: Lexique pratique du munjuk des rizières. Dialecte de Pouss. Paris, 1991., Librairie Orientaliste Paul

ÜKAPT I-VI = Sethe, K.: Übersetzung und Kommentar zu den altägyptischen Pyramidentexten. I-VI. Glückstadt, Hamburg, 1935-62., J. J. Augustin.

Vergote, J.: Review of Erman, A. \& Grapow, H.: Wörterbuch der ägyptischen Sprache, Band VI.= Muséon 63 (1950), 289-294.

VT $=$ Vetus Testamentum (Leiden).

Vycichl, W.: Hausa und Ägyptisch. Ein Beitrag zur historischen Hamitistik.= Mitteilungen des Seminars für Orientalische Sprachen an der Friedrich-Wilhelms-Universität zu Berlin 37 (1934), 36-116.

$\mathrm{Wb}=$ Erman, A. \& Grapow, H.: Wörterbuch der ägyptischen Sprache. I-V. ${ }^{2}$ Berlin, 1957-1971., Akademie-Verlag.

Westendorf, W.: Grammatik der medizinischen Texte. Berlin, 1962., Akademie-Verlag.

WMT $=$ Deines, H. von \& Westendorf, W.: Wörterbuch der medizinischen Texte. I-II. Berlin, 1961-2., Akademie-

Verlag.

ZÄS = Zeitschrift für Ägyptische Sprache und Altertumskunde (Leipzig).

Zyhlarz, E.: Konkordanz ägyptischer und libyscher Verbalstammtypen.= Zeitschrift für Ägyptische Sprache 70
(1934), 107-122. 